\title{
Sterile neutrinos with altered dispersion relations revisited
}

\author{
G. Barenboim, ${ }^{a, b}$ P. Martínez-Miravé, ${ }^{a, b}$ C.A. Ternes ${ }^{a}$ and M. Tórtola ${ }^{a, b}$ \\ ${ }^{a}$ Instituto de Física Corpuscular, CSIC-Universitat de València, \\ C/Catedrático José Beltrán 2, Paterna 46980, Spain \\ ${ }^{b}$ Departament de Física Teòrica, Universitat de València, \\ C/ Dr. Moliner 50, Burjassot 46100, Spain \\ E-mail: Gabriela.Barenboim@uv.es, pamarmi@ific.uv.es, \\ chternes@ific.uv.es, mariam@ific.uv.es
}

ABSTRACT: In this paper we investigate neutrino oscillations with altered dispersion relations in the presence of sterile neutrinos. Modified dispersion relations represent an agnostic way to parameterize new physics. Models of this type have been suggested to explain global neutrino oscillation data, including deviations from the standard three-neutrino paradigm as observed by a few experiments. We show that, unfortunately, in this type of models new tensions arise turning them incompatible with global data.

Keywords: Beyond Standard Model, Neutrino Physics

ARXIV EPRINT: 1911.02329 


\section{Contents}

1 Introduction 1

2 Altered dispersion relations in a $3+1$ scenario 2

2.1 Intrinsic modified dispersion relation 3

2.2 Modified dispersion relations from effective potentials 5

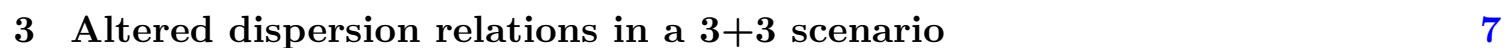

3.1 A resonant explanation for MiniBooNE avoiding reactor constraints $\quad 10$

4 Conclusions $\quad 12$

\section{Introduction}

Over the last approximately 20 years, neutrino oscillation measurements have become more and more precise and are now entering the precision era. Most of the current data coming from experiments using neutrinos from the Sun, reactors, the atmosphere and particle accelerators can be described in terms of three-neutrino oscillations, which depend on six oscillation parameters: two mass splittings $\left(\Delta m_{31}^{2}, \Delta m_{21}^{2}\right)$, three mixing angles $\left(\theta_{12}, \theta_{13}\right.$ and $\left.\theta_{23}\right)$ and a CP-violating phase $(\delta)$. Many of these parameters are measured very well as of now [1]. The remaining unknowns in this picture are the exact value of the CP-phase $\delta$, the octant of the atmospheric angle $\left(\sin ^{2} \theta_{23}<0.5\right.$ or $\left.\sin ^{2} \theta_{23}>0.5\right)$ and the neutrino mass ordering $\left(\Delta m_{31}^{2}>0\right.$ or $\left.\Delta m_{31}^{2}<0\right)$. The most recent oscillation data already provide some hints in favor of maximal CP violation and second octant of $\theta_{23}$, as well as a clear preference (above the $3 \sigma$ level) for the normal mass ordered neutrino spectrum [1], although they are not fully conclusive yet. Note that, combining oscillation data with recent cosmological observation results, a $3.5 \sigma$ preference for normal ordering can be obtained $[2,3]$.

Beyond the standard three-neutrino scenario, currently well established and characterized, some observations might suggest the existence of a fourth neutrino mass eigenstate. In the 90s, the LSND experiment observed the appearance of electron antineutrinos in a muon antineutrino beam [4-6]. A similar signal was recently observed in the MiniBooNE experiment [7]. Anomalies have also been observed in the electron (anti-) neutrino disappearance channel, known as the Gallium anomaly [8-11] and the reactor antineutrino anomaly [12]. The common feature of all these anomalous results is their short baseline, or $L / E$ of order $1 \mathrm{~km} / \mathrm{GeV}$ and, therefore, all of them can be explained in terms of a fourth sterile neutrino with $\Delta m_{41}^{2} \approx 1 \mathrm{eV}^{2}$, see for example ref. [13]. However, with new data coming from different long baseline experiments [14-18] a tension between the results observed in muon neutrino beams at disappearance and appearance channel arises, see refs. $[19,20]$. The reason is that the mixing angles and mass splittings required to explain 
the short baseline anomalies should produce a visible effect at the long baseline sector, that is absent. Therefore, the simplest $3+1$ scheme can not explain all the data simultaneously. For current reviews on this topic see refs. [21, 22]. It has been shown that adding simply more sterile neutrinos will not resolve this tension either [23].

This hot topic has been addressed in many articles since the latest results from MiniBooNE appeared [24-34]. As one can see, many theories are being tested, some of which are directly related to neutrino oscillations as in refs. [28, 29, 32-34].

In this paper, we focus on neutrino oscillations with altered dispersion relations (ADR) $[35,36]$. Modified dispersion relations are an economic and agnostic way to encompass a whole bunch of new physics models. Using the fact that neutrino oscillation experiments are nothing but a fancy interferometer (neutrinos are produced as flavour eigenstates but propagate as mass eigenstates), we can use them to study effects that would be otherwise too small to be observed, like Lorentz violation. ${ }^{1}$ As it is well known in the Standard Model, the (scalar) Higgs field acquires a vacuum expectation value (vev) breaking the electroweak symmetry and giving masses to fermions. Therefore, it won't be surprising that in string theory (or in quantum gravity) not a scalar but a tensor field would be the one acquiring a vev. As a result, the interaction of the fields that couple to these vev, which can be thought of as background fields, will be velocity and direction dependent. In other words, these vev will trigger the breakdown of Lorentz symmetry.

Of course, Lorentz violations can arise naturally also in theories with extra dimensions [37-40]. In this type of theories [35, 41, 42], sterile neutrinos can travel through the extra dimensions, causing a resonant oscillation behavior for a certain energy range, which might give an explanation for the anomalies observed in a few experiments [43, 44], without getting into conflict with cosmological observations [45], which is not the case for a scenario with simple sterile neutrinos [46]. The resonant behavior is a key ingredient in the set-up, as it allows to tune the energy range where the effect triggers and guarantees that it is set-off outside of this range. It has been argued [27], that these models do not affect the results obtained by the long baseline experiments. However, here we show that the parameters needed to produce sizeable effects in short baseline oscillations, indeed do spoil the oscillation probabilities in other neutrino oscillation experiments and, therefore, do not give a solution to the tension observed in short baseline oscillations.

Our paper is structured as follows: in section 2 we first give a brief introduction to $3+1$ mixing. Then, we discuss ADRs in this scenario, where we consider intrinsic ADRs and ADRs coming from an effective potential affecting neutrino propagation. In section 3 we extend this discussion to the case of three sterile neutrinos and address the consistency of its predictions. Finally, in section 4 we draw our conclusions.

\section{Altered dispersion relations in a $3+1$ scenario}

In order to explain the anomalies mentioned in the introduction, the existence of a fourth neutrino was suggested. This additional neutrino must be sterile, hence a Standard Model

\footnotetext{
${ }^{1}$ Let us remind the reader that, even if modified dispersion relations imply that Lorentz symmetry is broken, the theory is invariant under changes of coordinates.
} 
gauge singlet, or heavy enough to avoid bounds by LEP on the number of active neutrino families [47]. In this paper, we will consider only light sterile neutrinos. In this case, the lepton mixing matrix has to be extended, adding three new angles, two new phases and a new mass splitting. In the simplest scenario, the Hamiltonian describing the neutrino propagation in matter is given by

$$
H=\frac{1}{2 E} U\left(\begin{array}{cccc}
m_{1}^{2} & 0 & 0 & 0 \\
0 & m_{2}^{2} & 0 & 0 \\
0 & 0 & m_{3}^{2} & 0 \\
0 & 0 & 0 & m_{4}^{2}
\end{array}\right) U^{\dagger}+\left(\begin{array}{cccc}
V_{\mathrm{CC}} & 0 & 0 & 0 \\
0 & 0 & 0 & 0 \\
0 & 0 & 0 & 0 \\
0 & 0 & 0 & -V_{\mathrm{NC}}
\end{array}\right)
$$

Here, $E$ is the neutrino energy, $U$ is the matrix describing neutrino mixing, $m_{i}$ are the neutrino masses and $V_{\mathrm{CC}}$ and $V_{\mathrm{NC}}$ are the charged current $(\mathrm{CC})$ and neutral current (NC) potentials, respectively. Note that, since sterile neutrinos do not feel the weak interaction, the neutral current component of the potential, $V_{\mathrm{NC}}$, can not be eliminated from the expression of the effective potential, as it happens in the standard three-neutrino case. The neutrino mixing is now parameterized in terms of the $4 \times 4$ unitary matrix

$$
U=\tilde{U}_{34} U_{24} \tilde{U}_{14} U_{23} \tilde{U}_{13} U_{12}
$$

where the matrix $U_{i j}$ represents a rotation in the $i-j$ plane and the tilde indicates that the corresponding mixing angle is accompanied by a CP-phase. Since in this work we are interested in effects occurring only on short baselines or in channels which are not sensitive to matter effects, we will not consider the matter potential here. As argued in the introduction, this simplest extension can not explain the anomalous results obtained by a few experiments without being in tension with other experiments. In this section we consider two possible extensions of this model.

\subsection{Intrinsic modified dispersion relation}

The excess of events found in MiniBooNE can be studied assuming the existence of a sterile neutrino with $\Delta m_{41}^{2} \simeq 1 \mathrm{eV}^{2}$. In that case, the electron neutrino appearance probability is given by

$$
P_{\mu e} \simeq \sin ^{2} \theta_{24} \sin ^{2} 2 \theta_{14}\left(\frac{\Delta m_{41}^{2} L}{4 E}\right) .
$$

The existing bounds on $\theta_{24}$ come mainly from the non-observation of a signal of sterile neutrinos in the disappearance channel in MINOS/MINOS+ [14, 48] and IceCube [15]. At first approximation, the disappearance $\nu_{\mu}$ oscillation probability in the $3+1$ scheme is given by

$$
P_{\mu \mu} \simeq 1-\sin ^{2} 2 \theta_{23} \cos 2 \theta_{24} \sin ^{2}\left(\frac{\Delta m_{31}^{2} L}{4 E}\right)-\sin ^{2} 2 \theta_{24} \sin ^{2}\left(\frac{\Delta m_{41}^{2} L}{4 E}\right) .
$$

In the case of MINOS/MINOS+ and IceCube, since the kinematic phase $\frac{\Delta m_{41}^{2} L}{4 E}$ is very large, the last term in the expression above is considered to be averaged to $1 / 2 \sin ^{2} 2 \theta_{24}$. 
The strong bounds on the $3+1$ scenario coming from these experiments undermine the explanation of the anomalies in terms of a sterile neutrino. However, it has been claimed that altered dispersion relations could relax the tension between appearance and disappearance experiments.

A modification of the dispersion relation occurs when the energy momentum relation $E^{2}=p^{2}+m^{2}$ does not hold any more. Alterations of this type can appear in theories with Lorentz violation [49-52]. Here we will assume a generic Lorentz violating term associated to the fourth mass eigenstate. In this case, the kinematic phase changes according to

$$
\phi_{4 i}=\frac{\Delta m_{4 i}^{2} L}{4 E} \longrightarrow \phi_{4 i}=\left(\frac{\Delta m_{4 i}^{2}}{4 E}+f(E)\right) L, \quad \text { with } \quad i=1,2,3 .
$$

For simplicity, we choose $f(E)=\alpha E$. If the function $f(E)$ is positive $(\alpha>0)$, the kinematic phase is larger than its corresponding value in the $3+1$ neutrino standard framework, as it is shown in figure 1. This translates in the fact that probability terms controlled by $\Delta m_{41}^{2}$ get smeared out at smaller energies. Such a behavior has no impact on the bounds set by MINOS/MINOS+ and IceCube on $\theta_{24}$, since the term depending on $\phi_{41}$ is already averaged to $1 / 2$. Adding a modified dispersion relation that makes the kinematic phase grow with the energy would only result on this term getting averaged to $1 / 2$ at a lower energy. If the function $f(E)$ were negative $(\alpha<0)$, the kinematic phase could eventually reach very small values and even get to zero. In that case,

$$
P_{\nu_{\mu} \rightarrow \nu_{\mu}} \simeq 1-\sin ^{2} 2 \theta_{23} \cos 2 \theta_{24} \sin ^{2}\left(\frac{\Delta m_{31}^{2} L}{4 E}\right)-\sin ^{2} 2 \theta_{24} \phi_{41}^{2} .
$$

Then, we can conclude that a very small kinematic phase $\phi_{41}$ along the energy range of MINOS could weaken the bounds on $\theta_{24}$, since the bound would no longer apply to $\frac{1}{2} \sin ^{2} 2 \theta_{24}$ but to $\sin ^{2} 2 \theta_{24} \phi_{41}^{2}$. Indeed, one can always choose a modified dispersion relation $f(E)$ such that, for a given energy $E_{0}$ in the spectrum of MINOS, $f\left(E_{0}\right)=\Delta m_{41}^{2} / 4 E_{0}$ and, then, $\phi_{41}\left(E_{0}\right)=0$. Thus, if, along the energy spectrum of MINOS, $\phi_{41}$ were very small, it would be possible to weaken its bounds on the $3+1$ framework, as it was previously explained. However, the condition of $\phi_{41}$ being small would be valid only for a small interval of the energy spectrum. After a certain value of the energy, the modulus of the kinematic phase $\phi_{41}$ becomes very large and the $3+1$ neutrino picture is recovered, with a contribution from the sterile neutrino to the appearance probability equal to $1 / 2 \sin ^{2} 2 \theta_{24}$. This behavior is illustrated in figure 2. Different parametrisations of the modified dispersion relation $f(E)$ with physically reasonable energy dependencies have been explored. However, no substantial difference in the argumentation merits to be reported. Therefore, modified dispersion relations whose origin is the violation of Lorentz invariance, together with sterile neutrinos, can not reconcile the LSND and MiniBooNE anomalies with the results of other atmospheric and long baseline experiments, since the tension reported in refs. $[19,20]$ is not alleviated. Note that, apart from the problems mentioned here, neutrinos in this scenario would be superluminal, giving rise to further problems not discussed in this paper. 

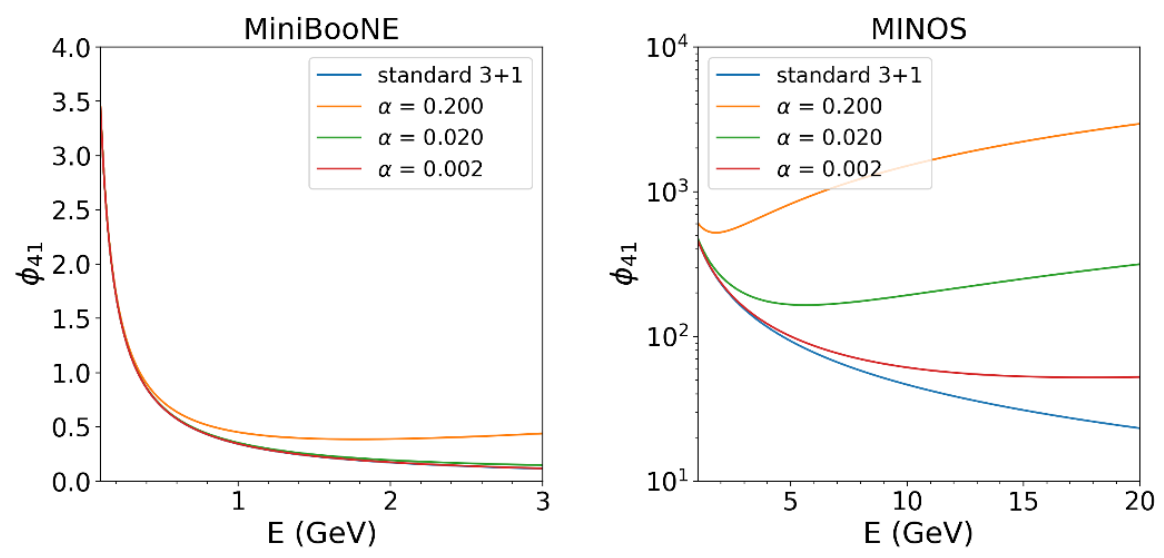

Figure 1. The kinematic phase $\phi_{41}$ as a function of the energy for MiniBooNE ( $\left.L=0.541 \mathrm{~km}\right)$ and MINOS $(L=731 \mathrm{~km})$ for $\Delta m_{41}^{2}=1.4 \mathrm{eV}^{2}$. Different values of $\alpha$ are also presented.
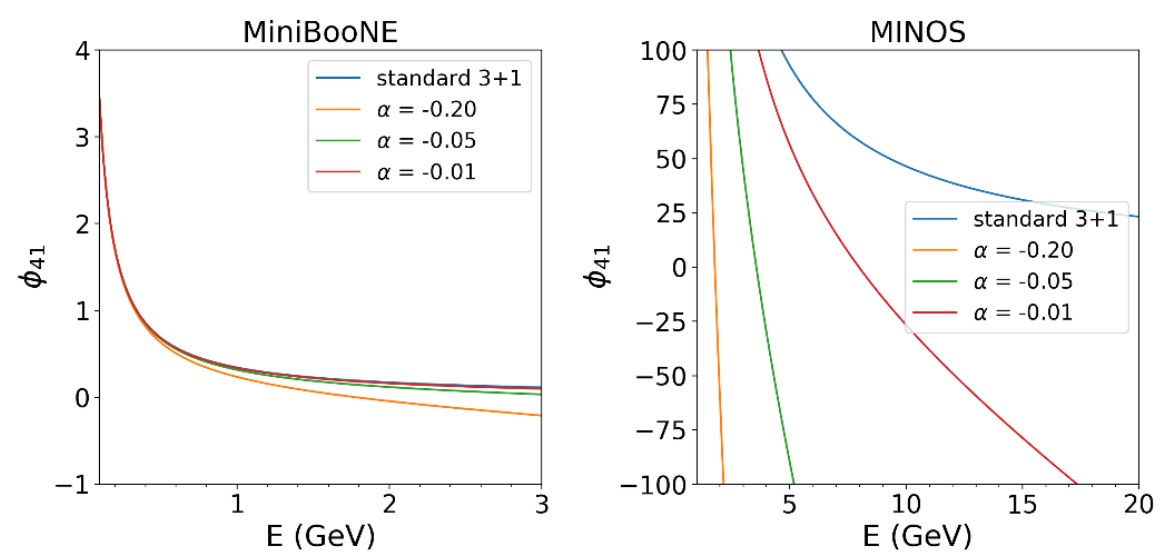

Figure 2. The kinematic phase $\phi_{41}$ as a function of the energy for MiniBooNE ( $\left.L=0.541 \mathrm{~km}\right)$ and MINOS $(L=731 \mathrm{~km})$. Different values of $\alpha$ are also presented.

\subsection{Modified dispersion relations from effective potentials}

Altered dispersion relations due to effective potentials in the Hamiltonian can lead to energy dependent oscillation parameters. The nature of such an additional potential can be shortcuts through extra dimensions [35, 42]. This type of modification, together with the existence of one or more sterile neutrinos, has been suggested as a solution to the anomalies found in neutrino oscillation experiments [27, 42]. In the $3+1$ scenario with modified dispersion relations, the neutrino Hamiltonian in vacuum is given by

$$
H=\frac{1}{2 E} U\left(\begin{array}{cccc}
m_{1}^{2} & 0 & 0 & 0 \\
0 & m_{2}^{2} & 0 & 0 \\
0 & 0 & m_{3}^{2} & 0 \\
0 & 0 & 0 & m_{4}^{2}
\end{array}\right) U^{\dagger}-\left(\begin{array}{cccc}
0 & 0 & 0 & 0 \\
0 & 0 & 0 & 0 \\
0 & 0 & 0 & 0 \\
0 & 0 & 0 & \epsilon E^{\alpha}
\end{array}\right)
$$


This type of effective potential was initially proposed for $\alpha=1$, which would correspond to sterile neutrinos traveling through extra dimensions [27,35]. The parameter $\epsilon$ is related to the time difference between the active and the sterile neutrino traveling through extra dimensions.

It is clear that such a potential induces energy dependencies in the oscillation parameters. The value of the parameter $\alpha$, which is model dependent, sets how wide or narrow the resonant effect is. It is important to notice that both mixing angles and mass splittings are now energy dependent. The latter ones can be obtained from the eigenvalues of the Hamiltonian, $\lambda_{i}$, as $m_{i \text {,eff }}^{2}=2 E \lambda_{i}(E)$. In principle, the resonant behavior of this scenario could relax the tension in data coming from appearance (MiniBooNE, essentially) and disappearance experiments (principally MINOS/MINOS+). In order to be consistent with ref. [27], we adopt the untypical parameterization

$$
U=U_{23} U_{13} U_{12} U_{14}
$$

In this notation only one mixing angle is needed to induce a non-zero short baseline appearance channel and not two mixing angles as in the standard case. The electron appearance probability in MiniBooNE is given by

$$
P_{\mu e} \simeq 4\left|U_{e 4}^{\mathrm{eff}}\right|^{2}\left|U_{\mu 4}^{\mathrm{eff}}\right|^{2} \sin ^{2}\left(\frac{\Delta m_{41}^{2, \mathrm{eff}} L}{4 E}\right),
$$

where $U^{\text {eff }}$ and $\Delta m_{\text {eff }}^{2}$ are the corresponding effective mixing matrix and mass splitting once the additional effective potential is considered. Therefore, if the combination $4\left|U_{e 4}^{\text {eff }}\right|^{2}\left|U_{\mu 4}^{\text {eff }}\right|^{2}$ happens to be large at the energy of the MiniBooNE anomalous signal $(E \leq 0.3 \mathrm{GeV})$, this mechanism could give rise to a significant appearance probability in MiniBooNE. Unfortunately, forcing $4\left|U_{e 4}^{\text {eff }}\right|^{2}\left|U_{\mu 4}^{\text {eff }}\right|^{2}$ to be large also affects the oscillation probabilities at long baseline experiments. In the upper left panel of figure 3 we plot the oscillation probability for MiniBooNE showing the required resonance at the energies of interest, as indicated by the blue curve. However, in the case of MINOS/MINOS+ (lower left panel) we see new fast oscillations, which should in average lower the signal rate in the disappearance channel with respect to the standard case (black line). The same happens in the disappearance channel at the T2K experiment. Most striking, however, is the expected signal at the appearance channel of T2K. There, one can see a very fast oscillation pattern reaching very large oscillation probabilities. This is due to the fact that the neutrino energy ranges covered by MiniBooNE and T2K overlap and, therefore, an energy dependent excess in MiniBooNE should have a visible effect in T2K as well. The standard oscillation parameters used to create these plots are those from table 1 . For the new parameters we choose $\sin ^{2} \theta_{14}=0.05, \Delta m_{41}^{2}=1.59 \mathrm{eV}^{2}$ and $\epsilon=5 \times 10^{-17} .{ }^{2}$ Note that using different values for $\delta$ would have no effect in MiniBooNE and leave also the T2K disappearance probability unchanged, while producing only a slight modification in the T2K appearance probability.

\footnotetext{
${ }^{2}$ We have checked different combinations of oscillation parameters, obtaining always the same qualitative result.
} 


\begin{tabular}{|c|c|}
\hline Parameter & Value \\
\hline$\Delta m_{21}^{2}$ & $7.55 \times 10^{-5} \mathrm{eV}^{2}$ \\
$\Delta m_{31}^{2}$ & $2.50 \times 10^{-3} \mathrm{eV}^{2}$ \\
$\sin ^{2} \theta_{12}$ & 0.32 \\
$\sin ^{2} \theta_{23}$ & 0.547 \\
$\sin ^{2} \theta_{13}$ & 0.0216 \\
$\delta$ & 0 \\
\hline
\end{tabular}

Table 1. The standard neutrino oscillation parameters used in the analysis, taken from ref. [1], except for $\delta$ which is set to zero for simplicity.

Previous studies [27] have pointed out an additional source of inconsistencies with the experimental data. In particular, it has been shown that, for energies above the resonance, and as a consequence of the energy dependence of the effective mass eigenstates, atmospheric neutrino experiments should also have presented clear deviations from the three-neutrino picture. Nonetheless, in a $3+3$ scheme this can be (unfortunately only) partially solved.

\section{Altered dispersion relations in a $3+3$ scenario}

Given the impossibility to reconcile the neutrino anomalous results in the context of a $3+1$ scenario with altered dispersion relations, some works have proposed an alternative explanation in terms of a $3+3$ scenario with or without extra new physics. If we consider three sterile neutrinos, our mixing matrix has to be further extended. The full mixing matrix is now given by [53]

$$
U=U_{36} U_{26} U_{16} U_{35} U_{25} U_{15} U_{34} U_{24} U_{14} U_{23} U_{13} U_{12},
$$

where we ignored possible CP-phases. The alteration of the dispersion relations can be introduced through an effective neutrino potential given by [27]

$$
V_{\text {eff }}=-\left(\begin{array}{cccccc}
0 & 0 & 0 & 0 & 0 & 0 \\
0 & 0 & 0 & 0 & 0 & 0 \\
0 & 0 & 0 & 0 & 0 & 0 \\
0 & 0 & 0 & \epsilon E & 0 & 0 \\
0 & 0 & 0 & 0 & \kappa E & 0 \\
0 & 0 & 0 & 0 & 0 & \xi E
\end{array}\right),
$$

where we introduce three new parameters $\epsilon, \kappa$ and $\xi$. This potential can be easily generalized by changing the power of the energy dependence. A resonant-like effect induced by this potential in MiniBooNE would require positive values for the coefficients $\epsilon, \kappa$ and $\xi$.

Note that the initial proposal of the model in ref. [27] uses an unconventional parametrization of the mixing matrix,

$$
U=U_{23} U_{13} U_{12} \hat{U}_{14} \hat{U}_{25} \hat{U}_{36},
$$



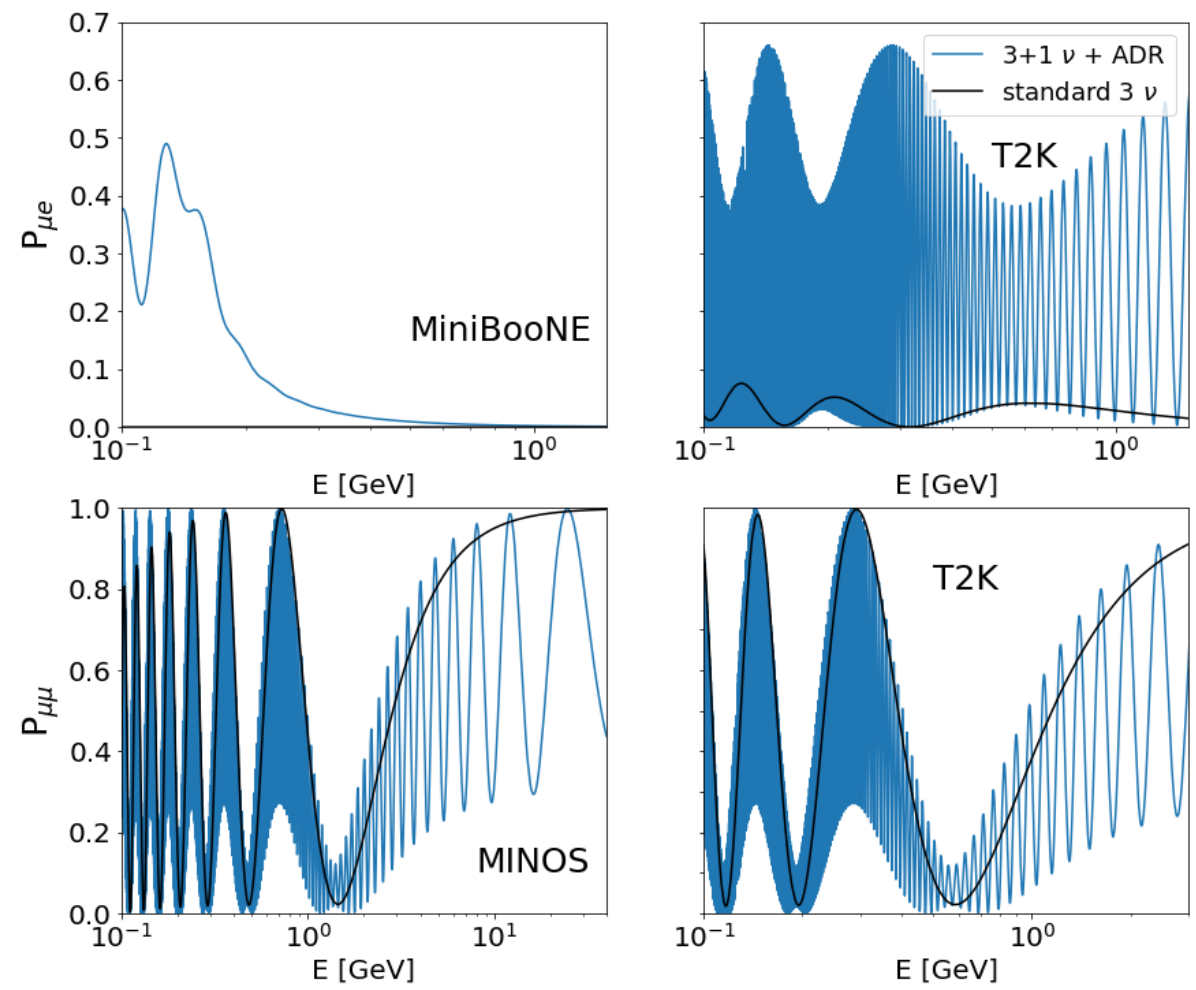

Figure 3. Comparison between the predictions of the three neutrino standard picture and a $3+1$ model with altered dispersion relations with $\epsilon=5 \times 10^{-17}$. The upper panels show the appearance probability at MiniBooNE $(L=0.541 \mathrm{~km})$ (left) and T2K $(L=295 \mathrm{~km})$ (right), while the lower panels show the disappearance probability at MINOS $(L=731 \mathrm{~km})$ (left) and T2K $(L=295 \mathrm{~km})$ (right).

with only three new mixing angles: $\hat{\theta}_{14}, \hat{\theta}_{25}, \hat{\theta}_{36}$. Moreover, they are imposed to be equal, $\hat{\theta}_{14}=\hat{\theta}_{25}=\hat{\theta}_{36}=\theta$. The reason for this shall be explained below. Since the original parametrization [27] is easier to handle for the discussion we are going to present, we will use it from now on. For our numerical studies we will use again the standard oscillation parameters from table 1 and the new parameters from table 2 , for which we use two different sets. ${ }^{3}$ For simplicity, we have set all of the CP-phases to zero.

We choose $\Delta m_{41}^{2}=1.59 \mathrm{eV}^{2}$. The other new mass differences are chosen to be

$$
\begin{aligned}
& \Delta m_{51}^{2}=\Delta m_{41}^{2}+\Delta m_{21}^{2}, \\
& \Delta m_{61}^{2}=\Delta m_{41}^{2}+\Delta m_{31}^{2} .
\end{aligned}
$$

These choices made in the initial proposal can potentially help to deal with the inconsistencies related to the values of the mass splittings at energies above the resonance. The idea behind it is that, above the resonance, sterile and active neutrinos swap their roles and the active-to-sterile mixing is suppressed. Then, the mass differences $\Delta m_{54}^{2}$ and $\Delta m_{64}^{2}$

\footnotetext{
${ }^{3}$ Note that we did not restrict our analysis only to these two sets, but tried to cover all the possibilities leading to a significant signal in MiniBooNE and LSND. We found the general trend to be similar to the one presented here.
} 


\begin{tabular}{|c|c|c|}
\hline parameter & set 1 & set 2 \\
\hline$\Delta m_{41}^{2}$ & $1.59 \mathrm{eV}^{2}$ & $1.59 \mathrm{eV}^{2}$ \\
$\sin ^{2} \theta$ & 0.05 & 0.05 \\
$\epsilon$ & $8 \times 10^{-16}$ & $5 \times 10^{-15}$ \\
$\kappa$ & $4 \times 10^{-17}$ & $5 \times 10^{-17}$ \\
$\xi$ & $4 \times 10^{-17}$ & $5 \times 10^{-17}$ \\
\hline
\end{tabular}

Table 2. New oscillation parameters used in the analysis, with $\theta=\hat{\theta}_{14}=\hat{\theta}_{25}=\hat{\theta}_{36}$.

are the ones accounting for the oscillations measured experimentally, $\Delta m_{21}^{2}$ and $\Delta m_{31}^{2}$, respectively. Consequently, at high energies they have to be equal to $\Delta m_{21}^{2}$ and $\Delta m_{31}^{2}$. Choosing $\hat{\theta}_{14}=\hat{\theta}_{25}=\hat{\theta}_{36}$ is necessary in order not to spoil this behavior at high energies.

Unfortunately, the tension between T2K and MiniBooNE arising from the energy dependence of the mixing angles is still present in models with altered dispersion relations and three sterile neutrinos. As in the $3+1$ case, it is possible to achieve the desired resonant effect in MiniBooNE, see the upper left panel of figure 4. This time also the MINOS/MINOS+ probability reproduces the standard one much better, since the fast oscillations appear only for rather low energies. However, it is clear that, as in the case of the $3+1$ scenario, the oscillation probabilities at $\mathrm{T} 2 \mathrm{~K}$ are spoiled, as shown in the right panels of figure 4 .

Another problem arises in the calculation of the effective mass splittings. After diagonalizing the Hamiltonian, one can calculate the effective masses, $m_{i \text {,eff }}^{2}(E)=2 E \lambda_{i}(E)$, and their differences from the eigenvalues, $\lambda_{i}(E)$, which depend on the energy. The desired behavior regarding the mass splittings is the following:

1. For energies below the one for which the LSND anomaly happens, $E<E_{\mathrm{LSND}} \sim$ $10 \mathrm{MeV}$, the standard mass differences $\Delta m_{21}^{2}$ and $\Delta m_{31}^{2}$ must be recovered. Therefore, the three sterile neutrinos must be considerable heavier than the active ones.

2. For energies larger than the one for which the MiniBooNE excess is found, $E>$ $E_{\mathrm{MB}} \sim 300 \mathrm{MeV}$, the active and sterile neutrinos should decouple. In this range, $\Delta m_{64}^{2}$ and $\Delta m_{54}^{2}$ have to recover the values of $\Delta m_{31}^{2}$ and $\Delta m_{21}^{2}$, respectively. In these two regimes far away from the resonances, the active-sterile mixing angles must be small.

3. To explain the observed signals in LSND and MiniBooNE, mass splittings of $\sim 1 \mathrm{eV}^{2}$ are needed and the mixing angles have to be large.

The energy dependence of the mass differences in this particular model is presented in figure 5. The resonant behavior needed to generate a large mass splitting for the energy ranges in LSND and MiniBooNE would also effect the energy range covered by reactor experiments (as indicated by the shaded regions in the figure), in particular Daya Bay and KamLAND, which observe neutrinos with energies in the range of 1-10 MeV. Daya Bay 

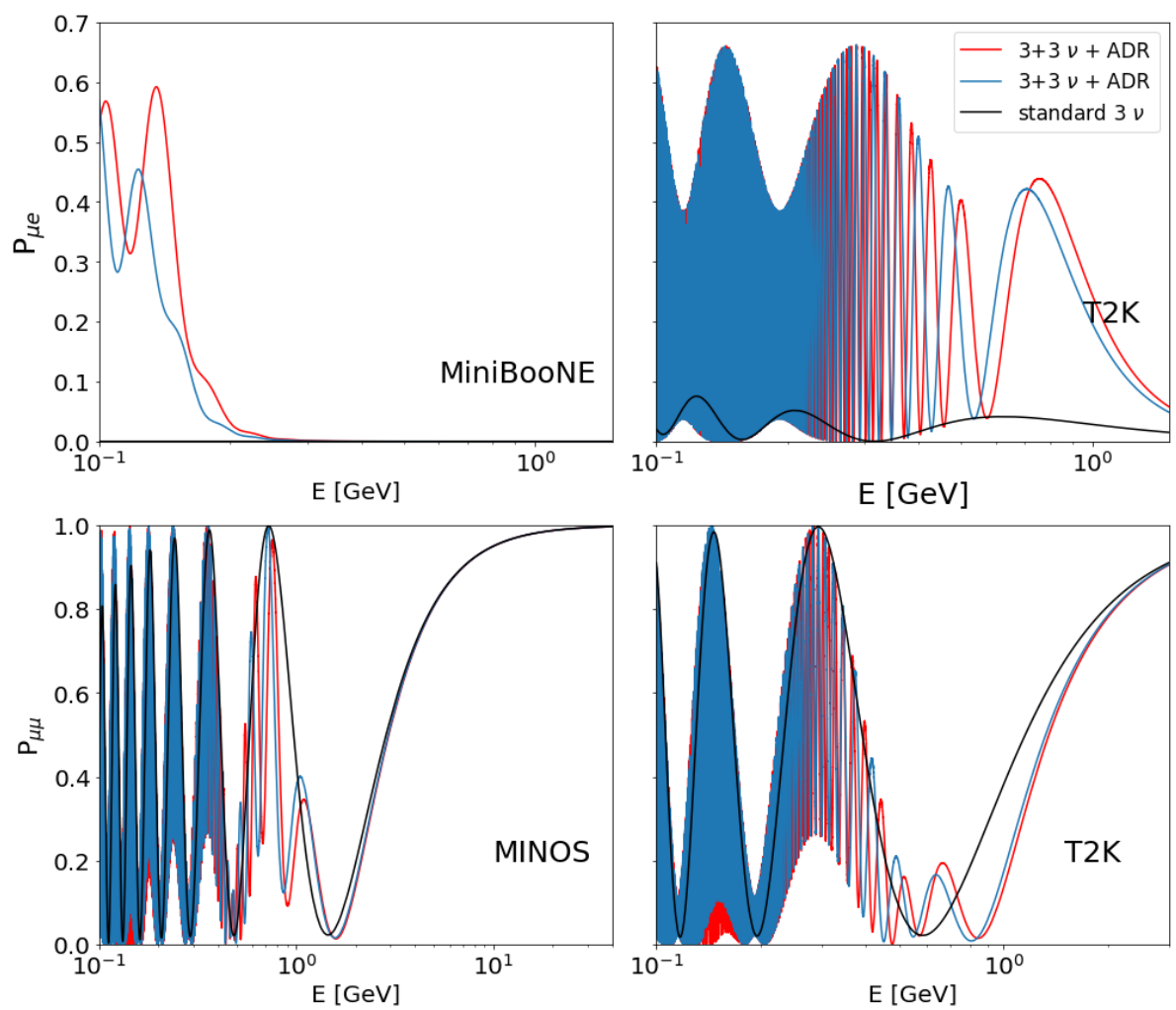

Figure 4. Comparison between the predictions of the three neutrino standard picture and a $3+3$ model with altered dispersion relations for the two sets of parameters mentioned in the main text.

can set strong constrains on this family of models, since its measurements of both $\theta_{13}$ and $\Delta m_{31}^{2}$ are very accurate [54]. KamLAND, on the other hand, measured $\Delta m_{21}^{2}$ with an excellent accuracy [55]. In this energy range, the dependence of the mass splittings on the energy is very relevant. As it is shown in figure 5 , the values of $\Delta m_{21}^{2}$ and $\Delta m_{31}^{2}$ predicted by the model differ significantly from the values measured at reactor experiments, namely $\Delta m_{21}^{2} \simeq 7.6 \times 10^{-5} \mathrm{eV}^{2}$ and $\Delta m_{31}^{2} \simeq 2.5 \times 10^{-3} \mathrm{eV}^{2}$. As a result, the predicted oscillation probabilities for Daya Bay and KamLAND deviate dramatically from the standard three neutrino framework, as one can see in figure 6 . Such a relevant deviation from the standard picture would have been easily detected already many years ago, so one can conclude that the model under study is not compatible with neutrino oscillation data. In the following subsection, however, we will discuss a very particular case where all the three new parameters are rather small. This choice moves the resonant behavior away from the energy range relevant to reactor neutrino experiments and, therefore, at low energies one recovers the effective 3+1 mixing. Hence, we can satisfactorily explain KamLAND and Daya Bay, since they are mostly unaffected by the $3+1$ mixing, as well as very short baseline reactor experiments and LSND. However, as we will see, the tensions with T2K will be still present.

\subsection{A resonant explanation for MiniBooNE avoiding reactor constraints}

As we have seen, the explanation of the MiniBooNE and LSND anomalous signals using resonant effects is in very strong tension with the well established reactor experiments. 


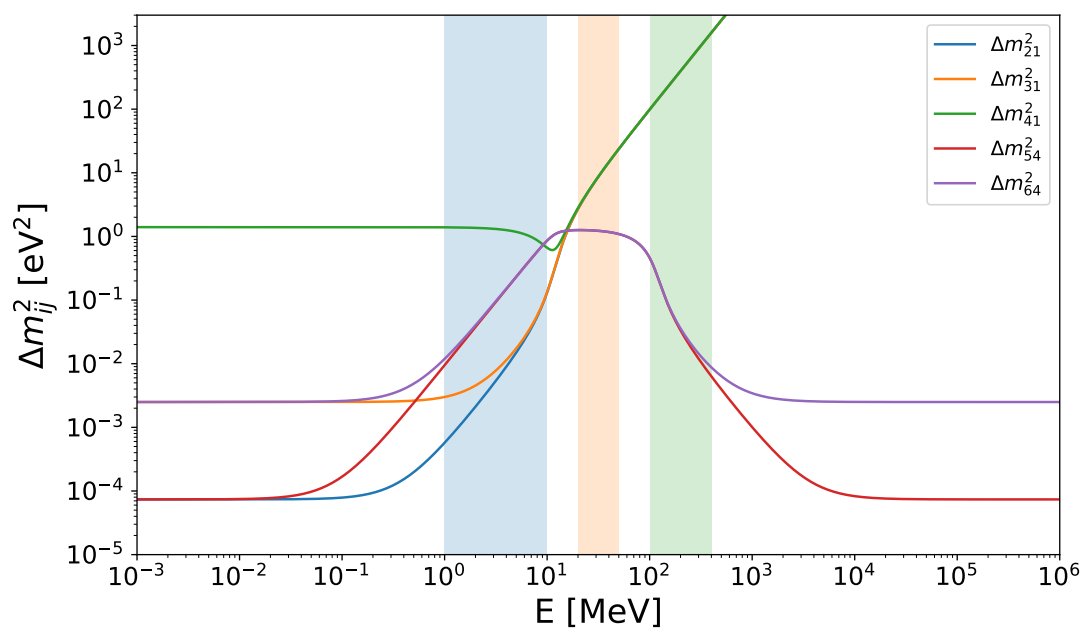

Figure 5. Effective mass splittings $\Delta m_{i j}^{2}$ as a function of the energy. The new parameters are fixed to the values from "set 2" in table 2. For the other set of parameters the picture looks very similar. The shaded region indicates the energy range relevant for reactor neutrino experiments (blue), LSND (orange) and MiniBooNE (green).
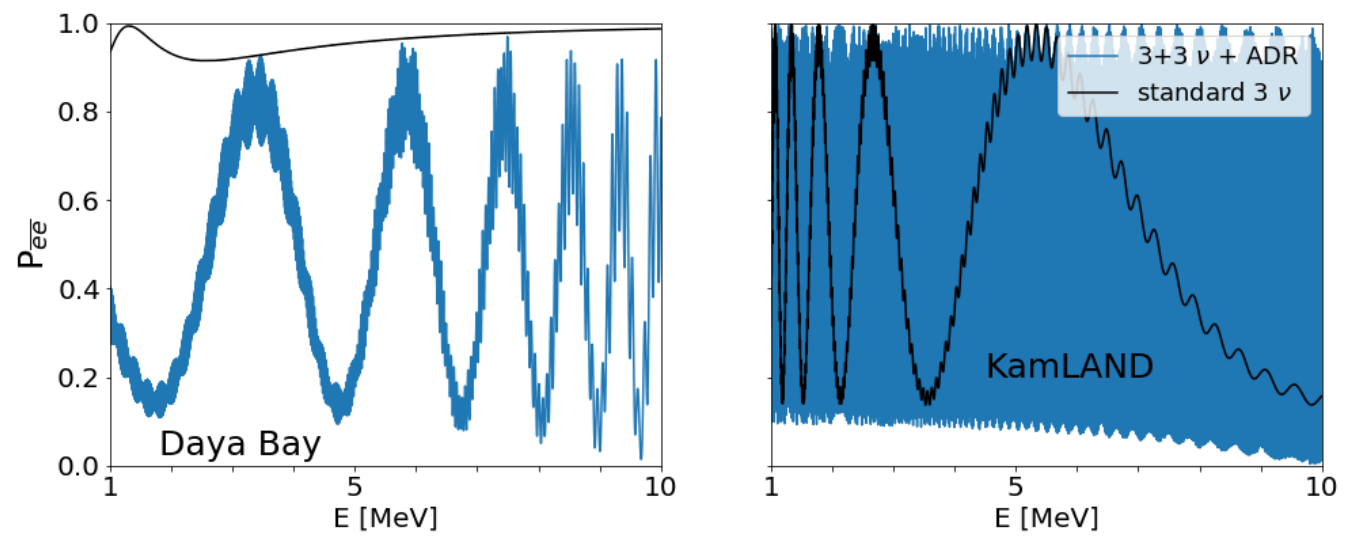

Figure 6. Comparison between the predictions of the three neutrino standard picture and the $3+3$ model with altered dispersion relations for Daya Bay (left) and KamLAND (right).

However, there is a conceptually interesting possibility that arises as a modification of the initial proposal [27]. If the three parameters in eq. (3.2) $(\epsilon, \kappa$ and $\xi)$ have similar values in a range such that the corresponding resonant energies lie in the region where the excess of events is found in MiniBooNE, one can avoid the inconsistencies with reactor experiments. This is due to the fact that, if the resonant effect happens at the order of $\mathcal{O}(0.1 \mathrm{GeV})$, the energy dependence of the mass splittings will not manifest in the energy range of the reactor experiments. The overall behavior in this particular case would be the following:

- For energies below $\sim 100 \mathrm{MeV}$, neutrino oscillations would be described by an effective $3+1$ picture. This allows to accommodate the LSND signal while being consistent with reactor experiments. 
- At $\sim 100-500 \mathrm{MeV}$, a resonant effect would account for the anomalous signal found in MiniBooNE.

- At higher energies, as it was discussed before, one would recover the three-neutrino picture once the parameters are chosen ad hoc to reproduce the experimental results. In this case, bounds from long baseline experiments would not apply directly to the parameters of the effective $3+1$ picture at low energies.

Nonetheless, predictions for experiments in the energy range between $100 \mathrm{MeV}$ and $10 \mathrm{GeV}$ are expected to be modified after considering these altered dispersion relations. The impact is expected to be particularly large in T2K, as previously shown. Deviations would appear in MINOS too, when the values of the parameters are chosen to explain the MiniBooNE signal with this mechanism, as is shown in figure 7. There, we show the predicted probabilities at MiniBooNE, MINOS and T2K for two different choices of parameters, as indicated in the caption. As can be seen in the figure, small deviations from this fine tuned scenario can also wreck the desired behavior of the oscillation probability in MiniBooNE, as indicated by the blue line. Note that this line in the MiniBooNE panel is systematically very close to zero and therefore this scenario would not create an excess. We show this scenario to highlight the instability of these solutions meant to avoid the reactor constraints and to show the level of fine-tuning needed to find them.

In addition to the discussion presented at the probability level, we have also calculated a $\chi^{2}$ value for our red benchmark point. Note that the blue benchmark point is already excluded by MiniBooNE, since it does not produce a sizeable oscillation probability there. This test has been performed using the same T2K data [56] and the same statistical analysis as in ref. [1]. To calculate the $\chi^{2}$ value, we marginalize over all the standard oscillation parameters relevant for T2K, namely $\Delta m_{31}^{2}, \sin ^{2} \theta_{23}, \sin ^{2} \theta_{13}$ and $\delta$. We obtain a value of $\chi^{2} \approx 237$, to be compared to the value in the standard neutrino oscillation scenario $\chi^{2} \approx$ 120, for 102 degrees of freedom. We should also remark that, in the scenario with ADRs, the best fit value for $\sin ^{2} \theta_{13}$ turns out to be very small. Then, if we include in our analysis a prior on $\sin ^{2} \theta_{13}$ coming from the Daya Bay measurement, the minimum $\chi^{2}$ value increases further to $\chi^{2} \approx 285$. Note that imposing a prior in this case is very well justified, since the new oscillation parameters were chosen to have no effect on reactor neutrino experiments. Thus, although our benchmark point would give rise to a significantly large probability that could potentially explain MiniBooNE, it is ruled out by T2K. A systematic search for points simultaneously compatible with the observed signals in MiniBooNE and T2K has produced no result. Indeed, a similar behavior to the one described above can be observed for any other point producing an observable oscillation probability at MiniBooNE: they are penalized with huge $\chi^{2}$ values in T2K. Therefore, one can conclude that this hypothesis does not provide a satisfactory explanation of neutrino oscillation data, including the anomalous LSND and MiniBooNE signals.

\section{Conclusions}

We have shown that an additional sterile neutrino satisfying an altered dispersion relation arising as a consequence of an effective potential can not give an explanation of the Mini- 

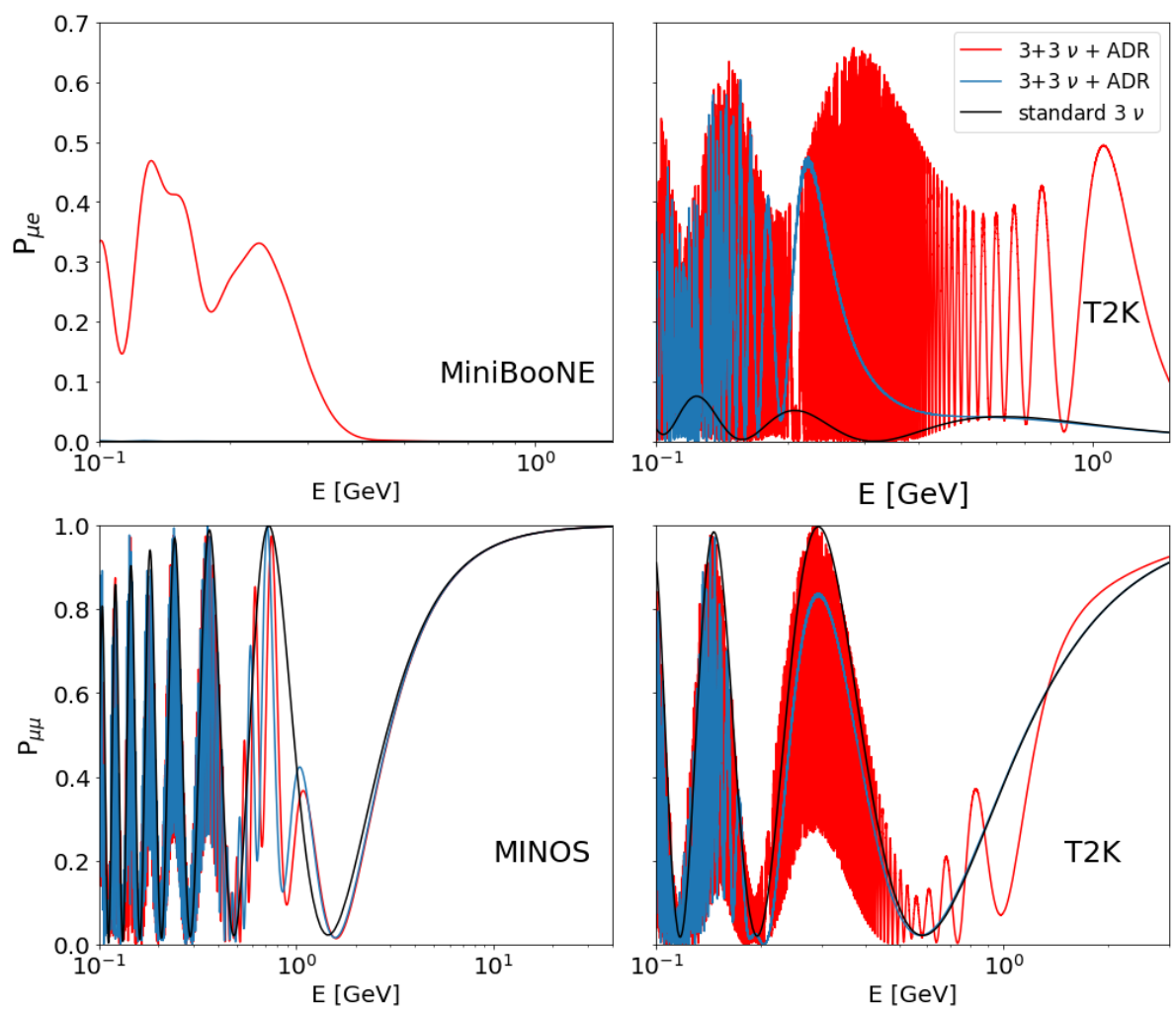

Figure 7. Comparison between the predictions of the three-neutrino standard picture (black) and a $3+3$ model with altered dispersion relations for $(\epsilon, \kappa, \eta)=(4.9,5,5) \times 10^{-17}$ (blue) and $(\epsilon, \kappa, \eta)=(1,5,5) \times 10^{-17}(\mathrm{red})$.

BooNE signal while, at the same time, being consistent with long baseline experiments, mainly MINOS/MINOS+ and T2K. Even in the case of more complex models with additional sterile neutrinos, the modification of the dispersion relation can not explain in a consistent picture current neutrino oscillation data and the observed anomalies. First, one finds that the resonant mixing angles required to explain the LSND and MiniBooNE excesses would have given rise to signals in other experiments, unobserved so far. Moreover, the dependence of the effective mass squared differences on the energy is strongly constrained by current reactor data and in disagreement with the predictions of this type of models. It is actually possible to avoid the constraints from reactor experiments if the resonant behavior is only invoked to explain the MiniBooNE anomalous signal. In this case, the lowest energy observables (essentially reactor and LSND data) will be described by an effective $3+1$ scenario, free of any further constraints from higher-energy experiments. Nevertheless, this proposal requires high levels of fine tuning and is very disfavoured by $\mathrm{T} 2 \mathrm{~K}$ results. Therefore, sterile neutrinos with altered dispersion relations can be added to a growing list of better or worse motivated physics that can not explain the anomalies observed in neutrino oscillation experiments. Should one come up with a model including any form of altered dispersion relations, these two energy-dependent effects have to be correctly addressed, since they would set strong constraints in the parameters of the model under study. 
Models of great complexity can be built in order to seek for an explanation to the anomalies in terms of sterile neutrinos. However, the number of parameters they require grows rapidly. The spirit that led to the proposal of oscillations with sterile neutrinos was to keep the explanation simple. If a large number of parameters was needed to phenomenologically explain the results from all the experiments, there would be no point on talking about sterile neutrino oscillations, since one would be eventually parametrizing some other physical phenomena. Therefore, greater efforts should be made in the search for explanations of the LSND and MiniBooNE signals which are not related to oscillations into sterile neutrinos.

As a parting remark, we would also like to mention that models with extra neutrinos can be seriously challenged by cosmological limits on the additional number of relativistic degrees of freedom, depending on the specifics of the dispersion relation. Likewise, a resonant mixing at the $\mathrm{MeV}$ scale can be severely compromised by BBN results.

\section{Acknowledgments}

We would like to thank Jorge Díaz and Heinrich Päs for useful comments and discussions. GB acknowledges support from the MEC and FEDER (EC) Grant SEV-2014-0398, FIS2015-72245-EXP, and FPA2017-845438 and the Generalitat Valenciana under grant PROMETEOII/2017/033, also partial support from the European Union FP10 ITN ELUSIVES (H2020-MSCAITN-2015-674896) and INVISIBLES-PLUS (H2020-MSCARISE2015-690575). PMM, CAT and MT are supported by the Spanish grants FPA2017-90566REDC (Red Consolider MultiDark), FPA2017-85216-P and SEV-2014-0398 (MINECO/ AEI/FEDER, UE), as well as PROMETEO/2018/165 (Generalitat Valenciana). PMM acknowledges financial support from MICINN through Programa Estatal de Fomento de la Investigación Científica y Técnica de Excelencia - Centros Severo Ochoa y Unidades María Maetzu - CSIC and the FPU grant FPU18/04571. CAT is supported by the FPI grant BES-2015-073593. PMM and CAT receive partial support from the EU Horizon 2020 project InvisiblesPlus (690575-InvisiblesPlus-H2020-MSCA-RISE-2015). PMM and CAT are grateful for the kind hospitality received at Fermilab during the final stage of this work. MT acknowledges financial support from MINECO through the Ramón y Cajal contract RYC-2013-12438.

Open Access. This article is distributed under the terms of the Creative Commons Attribution License (CC-BY 4.0), which permits any use, distribution and reproduction in any medium, provided the original author(s) and source are credited.

\section{References}

[1] P.F. de Salas, D.V. Forero, C.A. Ternes, M. Tortola and J.W.F. Valle, Status of neutrino oscillations 2018: $3 \sigma$ hint for normal mass ordering and improved CP sensitivity, Phys. Lett. B 782 (2018) 633 [arXiv:1708.01186] [InSPIRE].

[2] S. Gariazzo, M. Archidiacono, P.F. de Salas, O. Mena, C.A. Ternes and M. Tórtola, Neutrino masses and their ordering: Global Data, Priors and Models, JCAP 03 (2018) 011 [arXiv: 1801.04946] [INSPIRE]. 
[3] P.F. De Salas, S. Gariazzo, O. Mena, C.A. Ternes and M. Tórtola, Neutrino Mass Ordering from Oscillations and Beyond: 2018 Status and Future Prospects, Front. Astron. Space Sci. $\mathbf{5}$ (2018) 36 [arXiv:1806.11051] [INSPIRE].

[4] LSND collaboration, Evidence for neutrino oscillations from muon decay at rest, Phys. Rev. C 54 (1996) 2685 [nucl-ex/9605001] [INSPIRE].

[5] LSND collaboration, Evidence for $\nu_{\mu} \rightarrow \nu_{e}$ neutrino oscillations from LSND, Phys. Rev. Lett. 81 (1998) 1774 [nucl-ex/9709006] [INSPIRE].

[6] LSND collaboration, Evidence for neutrino oscillations from the observation of $\bar{\nu}_{e}$ appearance in a $\bar{\nu}_{\mu}$ beam, Phys. Rev. D 64 (2001) 112007 [hep-ex/0104049] [INSPIRE].

[7] MiniBoonE collaboration, Significant Excess of ElectronLike Events in the MiniBooNE Short-Baseline Neutrino Experiment, Phys. Rev. Lett. 121 (2018) 221801 [arXiv: 1805.12028] [INSPIRE].

[8] SAGE collaboration, Measurement of the solar neutrino capture rate with gallium metal. III: Results for the 2002-2007 data-taking period, Phys. Rev. C 80 (2009) 015807 [arXiv:0901.2200] [INSPIRE].

[9] M. Laveder, Unbound neutrino roadmaps, Nucl. Phys. Proc. Suppl. 168 (2007) 344 [INSPIRE].

[10] M.A. Acero, C. Giunti and M. Laveder, Limits on $\nu_{e}$ and $\bar{\nu}_{e}$ disappearance from Gallium and reactor experiments, Phys. Rev. D 78 (2008) 073009 [arXiv:0711.4222] [INSPIRE].

[11] C. Giunti and M. Laveder, Statistical Significance of the Gallium Anomaly, Phys. Rev. C 83 (2011) 065504 [arXiv: 1006. 3244] [INSPIRE].

[12] G. Mention et al., The Reactor Antineutrino Anomaly, Phys. Rev. D 83 (2011) 073006 [arXiv: 1101.2755] [INSPIRE].

[13] S. Gariazzo, C. Giunti, M. Laveder, Y.F. Li and E.M. Zavanin, Light sterile neutrinos, J. Phys. G 43 (2016) 033001 [arXiv: 1507.08204] [INSPIRE].

[14] MINOS+ collaboration, Search for sterile neutrinos in MINOS and MINOS+ using a two-detector fit, Phys. Rev. Lett. 122 (2019) 091803 [arXiv:1710.06488] [INSPIRE].

[15] ICECube collaboration, Searches for Sterile Neutrinos with the IceCube Detector, Phys. Rev. Lett. 117 (2016) 071801 [arXiv: 1605.01990] [INSPIRE].

[16] ICECuBE collaboration, Search for sterile neutrino mixing using three years of IceCube DeepCore data, Phys. Rev. D 95 (2017) 112002 [arXiv: 1702.05160] [InSPIRE].

[17] ANTARES collaboration, Measuring the atmospheric neutrino oscillation parameters and constraining the $3+1$ neutrino model with ten years of ANTARES data, JHEP 06 (2019) 113 [arXiv: 1812.08650] [INSPIRE].

[18] T2K collaboration, Search for light sterile neutrinos with the T2K far detector Super-Kamiokande at a baseline of 295 km, Phys. Rev. D 99 (2019) 071103 [arXiv: 1902.06529] [INSPIRE].

[19] S. Gariazzo, C. Giunti, M. Laveder and Y.F. Li, Updated Global $3+1$ Analysis of Short-BaseLine Neutrino Oscillations, JHEP 06 (2017) 135 [arXiv:1703.00860] [INSPIRE].

[20] M. Dentler et al., Updated Global Analysis of Neutrino Oscillations in the Presence of eV-Scale Sterile Neutrinos, JHEP 08 (2018) 010 [arXiv: 1803.10661] [INSPIRE].

[21] C. Giunti and T. Lasserre, eV-scale Sterile Neutrinos, Ann. Rev. Nucl. Part. Sci. 69 (2019) 163 [arXiv: 1901.08330] [INSPIRE]. 
[22] S. Böser et al., Status of Light Sterile Neutrino Searches, Prog. Part. Nucl. Phys. 111 (2020) 103736 [arXiv: 1906.01739] [INSPIRE].

[23] C. Giunti and E.M. Zavanin, Appearance-disappearance relation in $3+N_{s}$ short-baseline neutrino oscillations, Mod. Phys. Lett. A 31 (2015) 1650003 [arXiv:1508.03172] [InSPIRE].

[24] E. Bertuzzo, S. Jana, P.A.N. Machado and R. Zukanovich Funchal, Dark Neutrino Portal to Explain MiniBooNE excess, Phys. Rev. Lett. 121 (2018) 241801 [arXiv:1807.09877] [INSPIRE].

[25] E. Bertuzzo, S. Jana, P.A.N. Machado and R. Zukanovich Funchal, Neutrino Masses and Mixings Dynamically Generated by a Light Dark Sector, Phys. Lett. B 791 (2019) 210 [arXiv: 1808.02500] [INSPIRE].

[26] P. Ballett, S. Pascoli and M. Ross-Lonergan, U(1)' mediated decays of heavy sterile neutrinos in MiniBooNE, Phys. Rev. D 99 (2019) 071701 [arXiv:1808.02915] [INSPIRE].

[27] D. Döring, H. Päs, P. Sicking and T.J. Weiler, Sterile Neutrinos with Altered Dispersion Relations as an Explanation for the MiniBooNE, LSND, Gallium and Reactor Anomalies, arXiv: 1808.07460 [INSPIRE].

[28] J. Liao, D. Marfatia and K. Whisnant, MiniBooNE, MINOS+ and IceCube data imply a baroque neutrino sector, Phys. Rev. D 99 (2019) 015016 [arXiv:1810.01000] [INSPIRE].

[29] P.B. Denton, Y. Farzan and I.M. Shoemaker, Activating the fourth neutrino of the $3+1$ scheme, Phys. Rev. D 99 (2019) 035003 [arXiv:1811.01310] [InSPIRE].

[30] J.R. Jordan, Y. Kahn, G. Krnjaic, M. Moschella and J. Spitz, Severe Constraints on New Physics Explanations of the MiniBooNE Excess, Phys. Rev. Lett. 122 (2019) 081801 [arXiv: 1810.07185] [INSPIRE].

[31] C.A. Argüelles, M. Hostert and Y.-D. Tsai, Testing New Physics Explanations of MiniBooNE Anomaly at Neutrino Scattering Experiments, Phys. Rev. Lett. 123 (2019) 261801 [arXiv: 1812.08768] [INSPIRE].

[32] A. de Gouvêa, O.L.G. Peres, S. Prakash and G.V. Stenico, On The Decaying-Sterile Neutrino Solution to the Electron (Anti)Neutrino Appearance Anomalies, arXiv:1911.01447 [INSPIRE].

[33] M. Dentler, I. Esteban, J. Kopp and P. Machado, Decaying Sterile Neutrinos and the Short Baseline Oscillation Anomalies, arXiv:1911.01427 [INSPIRE].

[34] C. Giunti, A. Ioannisian and G. Ranucci, A new analysis of the MiniBooNE low-energy excess, arXiv:1912.01524 [INSPIRE].

[35] H. Pas, S. Pakvasa and T.J. Weiler, Sterile-active neutrino oscillations and shortcuts in the extra dimension, Phys. Rev. D 72 (2005) 095017 [hep-ph/0504096] [INSPIRE].

[36] S. Hollenberg, O. Micu, H. Pas and T.J. Weiler, Baseline-dependent neutrino oscillations with extra-dimensional shortcuts, Phys. Rev. D 80 (2009) 093005 [arXiv:0906.0150] [InSPIRE].

[37] N. Arkani-Hamed, S. Dimopoulos and G.R. Dvali, The Hierarchy problem and new dimensions at a millimeter, Phys. Lett. B 429 (1998) 263 [hep-ph/9803315] [INSPIRE].

[38] I. Antoniadis, N. Arkani-Hamed, S. Dimopoulos and G.R. Dvali, New dimensions at a millimeter to a Fermi and superstrings at a TeV, Phys. Lett. B 436 (1998) 257 [hep-ph/9804398] [INSPIRE].

[39] N. Arkani-Hamed, S. Dimopoulos and G.R. Dvali, Phenomenology, astrophysics and cosmology of theories with submillimeter dimensions and TeV scale quantum gravity, Phys. Rev. D 59 (1999) 086004 [hep-ph/9807344] [INSPIRE]. 
[40] N. Arkani-Hamed, S. Dimopoulos, G.R. Dvali and J. March-Russell, Neutrino masses from large extra dimensions, Phys. Rev. D 65 (2001) 024032 [hep-ph/9811448] [INSPIRE].

[41] D. Marfatia, H. Pas, S. Pakvasa and T.J. Weiler, A model of superluminal neutrinos, Phys. Lett. B 707 (2012) 553 [arXiv:1112.0527] [INSPIRE].

[42] D. Döring and H. Päs, Sterile Neutrino Shortcuts in Asymmetrically Warped Extra Dimensions, Eur. Phys. J. C 79 (2019) 604 [arXiv:1808.07734] [inSPIRE].

[43] S. Hollenberg, O. Micu, H. Pas and T.J. Weiler, Explaining LSND using extra-dimensional shortcuts, AIP Conf. Proc. 1200 (2010) 952 [arXiv:0908.3986] [InSPIRE].

[44] S. Hollenberg, O. Micu and H. Pas, Explaining LSND and MiniBooNE using altered neutrino dispersion relations, Prog. Part. Nucl. Phys. 64 (2010) 193 [arXiv:0911.1018] [INSPIRE].

[45] E. Aeikens, H. Päs, S. Pakvasa and T.J. Weiler, Suppression of cosmological sterile neutrino production by altered dispersion relations, Phys. Rev. D 94 (2016) 113010 [arXiv: 1606.06695] [INSPIRE].

[46] S. Gariazzo, P.F. de Salas and S. Pastor, Thermalisation of sterile neutrinos in the early Universe in the $3+1$ scheme with full mixing matrix, JCAP 07 (2019) 014 [arXiv: 1905.11290] [INSPIRE].

[47] ALEPH, DELPHI, L3, OPAL, SLD collaborations, LEP Electroweak Working Group, SLD Electroweak Group and SLD Heavy Flavour Group, Precision electroweak measurements on the $Z$ resonance, Phys. Rept. 427 (2006) 257 [hep-ex/0509008] [INSPIRE].

[48] MINOS collaboration, Search for Sterile Neutrinos Mixing with Muon Neutrinos in MINOS, Phys. Rev. Lett. 117 (2016) 151803 [arXiv:1607.01176] [INSPIRE].

[49] V.A. Kostelecky and M. Mewes, Lorentz and CPT violation in neutrinos, Phys. Rev. D 69 (2004) 016005 [hep-ph/0309025] [INSPIRE].

[50] J.S. Diaz and A. Kostelecky, Lorentz- and CPT-violating models for neutrino oscillations, Phys. Rev. D 85 (2012) 016013 [arXiv:1108.1799] [InSPIRE].

[51] A. Kostelecky and M. Mewes, Neutrinos with Lorentz-violating operators of arbitrary dimension, Phys. Rev. D 85 (2012) 096005 [arXiv:1112.6395] [INSPIRE].

[52] G. Barenboim, M. Masud, C.A. Ternes and M. Tórtola, Exploring the intrinsic Lorentz-violating parameters at DUNE, Phys. Lett. B 788 (2019) 308 [arXiv:1805.11094] [INSPIRE].

[53] Z.-z. Xing, A full parametrization of the $6 \times 6$ flavor mixing matrix in the presence of three light or heavy sterile neutrinos, Phys. Rev. D 85 (2012) 013008 [arXiv:1110.0083] [INSPIRE].

[54] DaYA Bay collaboration, Measurement of the Electron Antineutrino Oscillation with 1958 Days of Operation at Daya Bay, Phys. Rev. Lett. 121 (2018) 241805 [arXiv:1809.02261] [INSPIRE].

[55] KamLAND collaboration, Precision Measurement of Neutrino Oscillation Parameters with KamLAND, Phys. Rev. Lett. 100 (2008) 221803 [arXiv: 0801.4589] [inSPIRE].

[56] T2K collaboration, Search for CP-violation in Neutrino and Antineutrino Oscillations by the T2K Experiment with $2.2 \times 10^{21}$ Protons on Target, Phys. Rev. Lett. 121 (2018) 171802 [arXiv: 1807.07891] [INSPIRE]. 